\title{
STRUCTURE-FUNCTION ANALYSIS OF PEROXIDASIN PROVIDES INSIGHT INTO THE MECHANISM OF COLLAGEN IV CROSSLINKING
}

Enikő Lázár ${ }^{1,2}$, Zalán Péterfi ${ }^{1,2}$, Gábor Sirokmány ${ }^{1,2}$, Hajnal A. Kovács ${ }^{1,2}$, Eva Klement ${ }^{3}$, Katalin F. Medzihradszky ${ }^{3,4}$ and Miklós Geiszt ${ }^{1,2}$

${ }^{1}$ Department of Physiology, Semmelweis University, Budapest, Hungary

2“"Momentum” Peroxidase Enzyme Research Group of the Semmelweis University and the Hungarian Academy of Sciences

${ }^{3}$ Laboratory of Proteomics Research, Institute of Biochemistry, Biological Research Centre of the Hungarian Academy of Sciences, Szeged, Hungary

${ }^{4}$ Department of Pharmaceutical Chemistry, University of California, San Francisco, USA

*Corresponding author: Miklós Geiszt

Department of Physiology, Semmelweis University, Faculty of Medicine, PO Box 259

H-1444 Budapest, Hungary

Telephone: 36-1-459-1500 ext: 60415

Fax: 36-1-266-7480

e-mail: geiszt@eok.sote.hu

Running title: Structure-function analysis of peroxidasin 


\section{ABSTRACT}

Basement membranes provide structural support and convey regulatory signals to cells in diverse tissues. Assembly of collagen IV into a sheet-like network is a fundamental mechanism during the formation of basement membranes. Peroxidasin (PXDN) was recently described to catalyze crosslinking of collagen IV through the formation of sulfilimine bonds. Despite the significance of this pathway in tissue genesis, our understanding of PXDN function is far from complete. In this work we demonstrate that collagen IV crosslinking is a physiological function of mammalian PXDN. Moreover, we carried out structure-function analysis of PXDN to get a better insight into its role in collagen IV synthesis. We identify conserved cysteines in PXDN which mediate the oligomerization of the protein into a trimeric complex. We also demonstrate that oligomerization is not an absolute requirement for enzymatic activity but optimal collagen IV coupling is only catalyzed by the PXDN trimers. Localization experiments of different PXDN mutants in two different cell models revealed that PXDN oligomers, but not monomers, adhere on cell-surface in "hot spots", which represent previously unknown locations of collagen IV crosslinking.

Keywords: collagen IV / crosslink / peroxidase / peroxidasin / reactive oxygen species

Abbreviations: PXDN: peroxidasin, PXDNL: peroxidasin-like protein, NC1 domain: noncollagenous domain, LRR: leucine-rich repeat, Ig C2: immunoglobulin-like C2 domain, vWF C: von Willebrand factor C-type domain, MEF: mouse embryonic fibroblast, TCL: total cell lysate, CDL: collagenase-digested lysate 


\section{INTRODUCTION}

Once considered ubiquitously toxic, reactive oxygen species (ROS) are now recognized as important effector and signaling molecules in living organisms. Among diverse forms of reactive oxygen, hydrogen peroxide $\left(\mathrm{H}_{2} \mathrm{O}_{2}\right)$ seems to be a particularly important molecule (1). $\mathrm{H}_{2} \mathrm{O}_{2}$ has direct regulatory effects on signaling networks through reversible cysteine oxidation, but the best understood effects of $\mathrm{H}_{2} \mathrm{O}_{2}$ are mediated by animal heme peroxidases (2).

The family of mammalian heme peroxidases consists of myeloperoxidase (MPO), eosinophil peroxidase (EPX), thyroid peroxidase (TPO), lactoperoxidase (LPO) and peroxidasin (PXDN). A peroxidasin-like protein (PXDNL) was recently identified in humans (3), however this protein lacks measurable peroxidase activity. Animal heme peroxidases (MPO, EPX and LPO) serve in the first line of host defense, while TPO has a crucial role in thyroid hormone synthesis. The idea that heme peroxidases participate in the formation of the extracellular matrix (ECM) of animals first gained support from experiments on sea urchin eggs, where stabilization of the fertilization envelope is partially mediated by ovoperoxidasecatalyzed crosslinking of tyrosine residues (4). ECM modification through dityrosine formation was also described to have a role in cuticle formation of C. elegans (5).

Compared to other members of the enzyme family peroxidasin is quite unique since besides its peroxidase domain it contains modules that are characteristic of constituents of the ECM $(6 ; 7)$. These domains include N-terminal leucine-rich repeats, immunoglobulin C2 domains and a vWF C-type domain at the very $\mathrm{C}$-terminus of the protein. The unique combination of peroxidase domain along with ECM motifs suggests a special role for PXDN in ECM formation. 
PXDN was first described in Drosophila and subsequent studies revealed that it is ubiquitously expressed across the animal kingdom (6;8-10). Although secretion of PXDN into the extracellular space was demonstrated in both Drosophila and primary mammalian cells, the physiological significance of its peroxidase activity was unknown for a long time. Recently Bhave et al. identified PXDN as the long-sought player in collagen IV biosynthesis that catalyzes crosslinking of collagen IV trimers through their NC1 domains (11). Coupling of the NC1 domains occurs through sulfilimine bonds that were only recently recognized in living organisms (12). While vast amount of data have been collected about the structure and enzymatic activity of other members of the animal heme peroxidase family (13), we know very little about the PXDN protein and the mechanism of collagen IV crosslinking. In this study we sought to determine the supramolecular architecture of PXDN and the structural determinants of its peroxidase activity and adhesive properties. In rescue experiments using embryonic fibroblasts prepared from PXDN-deficient animals we also determined the structural requirements of PXDN for collagen IV crosslinking. 


\section{MATERIALS AND METHODS}

\section{Generation of recombinant PXDN constructs}

cDNA encoding full-length human PXDN was directly inserted into pcDNA3.1/V5-HisTOPO vector using TOPO TA Expression Kit (Invitrogen, Life Technologies). Targeted mutations were introduced into the full-length sequence with double-primer PCR method using complementary mutagenic oligonucleotide pairs (Sigma-Aldrich). Mutated constructs were amplified with Phusion Hot Start II High-Fidelity DNA Polymerase (Thermo Scientific). To avoid the occurrence of any undesired mutations, DNA regions containing the targeted nucleotide changes were sequenced (Eurofins MWG Operon) and cloned into the original vector backbone using suitable restriction endonuclease pairs (Fermentas, Thermo Scientific). Truncated constructs were generated by inserting particular regions of PXDN coding sequence into pcDNA3.1/V5-His-TOPO vector using TA cloning strategy. We obtained cDNA of a previously described shorter PXDN isoform (14) by 5' and 3' RACE using poly(A) RNA of cadmium chloride-stimulated EB1 colon carcinoma cell line and used it to generate the N-termially truncated $\mathrm{PXDN}_{695-1479}$ construct. To create $\mathrm{PXDN}_{1-26+696-1479}$, oligonucleotides corresponding to the original secretory signal sequence (aa1-26) of PXDN were hybridized and then ligated between unique restriction sites following the start codon of PXDN $_{695-1479 .}$ Insert of the C-terminally truncated $\mathrm{PXDN}_{1-1412}$ construct was amplified with Phusion Hot Start II from the full-length PXDN sequence with specific PCR primers flanking the selected nucleotide region. Appropriate restriction fragments of $\mathrm{PXDN}_{1-26+696-1479}$ and PXDN $_{1-1412}$ were joined with T4 DNA Ligase (Thermo Scientific), thus forming PXDN $1-26+696-$ 1412. All coding regions synthesized in polymerase chain reactions were sequenced to rule out any infidelity between the template DNA and the PCR product. 
Empty pcDNA3.1/V5-His vector was routinely used as control for transfections in all the structural and functional assays. Schematic structures of the above mentioned recombinant PXDN constructs are summarized in Supplementary figure 1.

\section{Generation and breeding of PXDN knockout mice}

PXDN-deficient mice were generated by $\mathrm{SAGE}^{\circledR}$ Laboratories using CompoZr zinc finger endonuclease technology. Detailed characterization of PXDN-deficient animals is subject of a different paper. Timed breedings were set up between $\mathrm{Pxdn}^{+/-}$mice to generate wild-type and PXDN knockout embryos. Pregnant females were sacrificed by cervical dislocation 13 days post coitum, their uteri were dissected and the embryos were harvested for genotyping and preparation of mouse embryonic fibroblasts (MEFs).

\section{Cell culture and treatments}

HEK293 freestyle (Life Technologies) and Cos7 cells (ATCC) were cultured in Dulbecco's Modified Eagle's Medium (DMEM; Lonza) supplemented with 10\% fetal bovine serum (FBS; Lonza) and $100 \mathrm{U} / \mathrm{ml}$ penicillin/100 $\mu \mathrm{g} / \mathrm{ml}$ streptomycin (Lonza). Transient transfections were carried out with Lipofectamine LTX and Plus Reagents (Life Technologies) following the manufacturer's instructions. To establish a stable cell line constitutively expressing V5-tagged recombinant PXDN, we transfected HEK293 freestyle cells with pcDNA3.1/PXDN-V5-His construct containing neomycin resistance gene. To select stably-transfected cells, we completed the culture medium with $1000 \mu \mathrm{g} / \mathrm{ml}$ geneticin (G418 sulphate; Life Technologies) 48 hours after transfection and maintained the selection pressure for 6 weeks. Following isolation of surviving cells, several clones were tested for PXDN expression in Western blot experiments. Those with the highest PXDN levels were further cultivated using $400 \mu \mathrm{g} / \mathrm{ml}$ geneticin and were used for purification of the recombinant 
protein. Human umbilical vein endothelial cells (HUVECs; Lonza) were grown in EGM-2 medium (Lonza) to study oligomerization of endogenous PXDN. Mouse embryonic fibroblasts (MEFs) were isolated from wild-type (WT) and PXDN-deficient (KO) animals. Following the removal of the head and red tissues, remaining body wall of the embryos was finely minced and repeatedly incubated with $5 \mathrm{ml}$ 1x Trypsin/Versene (EDTA) (Lonza) at 37 ${ }^{\circ} \mathrm{C}$ for 10 minutes. After sedimentation of undigested tissues, supernatant was mixed with 40 $\mathrm{ml}$ freshly prepared medium consisting of DMEM, 10\% FBS, $100 \mathrm{U} / \mathrm{ml}$ penicillin $/ 100 \mu \mathrm{g} / \mathrm{ml}$ streptomycin, 1x MEM Non-Essential Amino Acid Solution (NEAA; Sigma-Aldrich), 1x MEM Vitamin Solution (Sigma-Aldrich) and $50 \mu \mathrm{g} / \mathrm{ml}$ ascorbic acid (Sigma-Aldrich). The cells were centrifuged at $377 \mathrm{x}$ g for 5 minutes, then the pellet was resuspended in fresh medium and was plated in sterile flasks. After their first passage MEFs were grown to $90 \%$ confluency when they were frozen in numerous vials for long-term storage. We used thawed P1 MEF colonies for experiments requiring this cell type. Transfection of PXDN mutant MEFs was carried out with electroporation (1 pulse, $1350 \mathrm{~V}, 30 \mathrm{~ms}$ ) using Neon Transfection System (Invitrogen). Cell cultures were maintained in a $37{ }^{\circ} \mathrm{C}$ incubator with humidified atmosphere of $5 \% \mathrm{CO}_{2}$ in air.

\section{Primary antibodies}

Polyclonal antibodies recognizing the C-terminal segment of human ( $\alpha$-hsPXDN, aa13291479) or mouse ( $\alpha-m m P X D N$, aa1326-1475) PXDN were raised in rabbits following repeated intracutaneous injections of GST-tagged peptides. Sera of the immunized animals were depleted of GST-specific antibodies and then were affinity-purified with Affigel 10 (Bio-Rad) coupled to PXDN peptides which were previously used for the immunization. Specificity of $\alpha-\mathrm{mmPXDN}$ was tested with knockout control in all the described applications while validation of $\alpha$-hsPXDN has already been reported in our previous paper (9). Monoclonal $\alpha-$ 
V5 antibody used for Western blot and immunostaining experiments was purchased from AbD Serotec. Mouse anti- $\beta$ actin antibody obtained from Sigma-Aldrich was routinely used as loading control in Western blot experiments. Rat monoclonal antibody against the NC1 domain of collagen IV alpha 2 isoform ( $\alpha$-coll4a2) was acquired from Chondrex, Inc..

\section{Analysis of PXDN oligmerization}

HUVECs and HEK293 freestyle cells expressing recombinant PXDN constructs were preincubated with 300 mM HEPES pH 7.4 containing 1 mM N-ethylmaleimide (NEM; Sigma-Aldrich) for 10 minutes, and then were lysed in RIPA buffer $(0.5 \%$ sodium deoxycholate (DOC; USB Corporation), $0.1 \%$ sodium dodecyl sulphate (SDS; Affymetrix), 1\% Triton X-100 (Sigma-Aldrich), $150 \mathrm{mM} \mathrm{NaCl,} 50 \mathrm{mM}$ Tris $\mathrm{pH}$ 8.0) supplemented with protease inhibitors (10 $\mu \mathrm{g} / \mathrm{ml}$ aprotinin (Sigma-Aldrich), $1 \mathrm{mM}$ phenylmethylsulfonyl fluoride (PMSF; Sigma-Aldrich), cOmplete Mini EDTA-Free Protease Inhibitor Cocktail Tablets (Roche)) and $1 \mathrm{mM}$ NEM. Lysates were spun at $16100 \mathrm{x}$ g for 10 minutes to remove insoluble cell fraction. Supernatants were then divided and mixed with 4x Laemmli sample buffer with or without $0.5 \% \beta$-mercaptoethanol ( $\beta \mathrm{ME}$; Sigma-Aldrich), then the reduced samples were boiled at $96{ }^{\circ} \mathrm{C}$ for 8 minutes. Non-reduced lysates were separated on $4 \%$ or $5 \%$ polyacrylamide (Bio-Rad)-SDS gels beside ProSieve QuadColor Protein Marker (4.6 kD-300 kD; Lonza) and HiMark Pre-Stained Protein Standard (Novex, Life Technologies). Oligomerization of the endogenous or recombinant proteins was evaluated based on the presence of PXDN- or V5-specific signals at molecular masses corresponding to trimeric, dimeric or monomeric PXDN in Western blots. Transfection efficiency of the examined recombinant constructs was compared in $\alpha-\mathrm{V} 5$ immunoblots of reduced samples.

\section{Detection of NC1 crosslinking}


Crosslinking activity of endogenously expressed PXDN was analyzed using wild-type and PXDN knockout mouse embryonic fibroblasts. MEFs grown for 4 days in 6-well culture plates were washed with $300 \mathrm{mM}$ HEPES pH 7.4 and then were scraped up in hypotonic lysis buffer (10 $\mathrm{mM} \mathrm{CaCl}_{2}, 50 \mathrm{mM}$ HEPES pH 7.4) completed with $0.1 \mathrm{mM}$ benzamidine hydrochloride, $1 \mathrm{mM}$ PMSF and $1 \mathrm{mM}$ NEM. One part of total cell lysates was boiled for 8 minutes at $96{ }^{\circ} \mathrm{C}$ with $4 \mathrm{x}$ Laemmli buffer and was used to confirm phenotype of the cells in PXDN Western blots. The rest of the cell lysates was digested with $0.5 \mathrm{mg} / \mathrm{ml}$ type I collagenase (Worthington) at $37^{\circ} \mathrm{C}$ for 24 hours. Following separation of the digests with SDS-PAGE, the proteins were blotted on nitrocellulose membranes and crossliked dimeric and uncrosslinked monomeric NC1 domains of collagen IV were detected with $\alpha$-coll4a2 antibodies. To investigate their potential of rescuing collagen IV crosslinking in the mutant cells, recombinant human PXDN constructs were transfected into PXDN-deficient MEFs. Expression of recombinant proteins was checked and compared in Western blots 24 hours after transfection, collagenase digestion of corresponding samples was started 3 days later to provide enough time for secretion and assembly of collagen IV network.

\section{Analysis of PXDN secretion}

Cos7 cells were seeded in 12-well cell culture plates and were transfected with several recombinant PXDN constructs right after attachment. 12 hours after transfection the DMEMbased culture medium was changed to protein-free Freestyle 293 Expression Medium (Life Technologies). The cell culture medium and transfected cells were both harvested after another 24 hours. First the medium was spun at $800 \mathrm{x}$ g for 10 minutes to avoid contamination with cell debris, then $4 \mathrm{x}$ Laemmli sample buffer was added to the supernatant and the samples were boiled for 8 minutes at $96{ }^{\circ} \mathrm{C}$. The cells were washed once with $300 \mathrm{mM}$ HEPES pH 7.4, 
then were scraped up in 4x Laemmli buffer. Following SDS-PAGE the cell lysates and medium samples were probed for PXDN and actin content in Western blots.

\section{Western blotting and immunoprecipitation}

Following gel electrophoresis of reduced or non-reduced samples, separated proteins were blotted on nitrocellulose membranes. Blots were blocked in PBS containing 0.1\% Tween (Sigma-Aldrich) and 5\% milk powder or 5\% bovine serum albumin (BSA; Affymetrix) and then were incubated with the primary antibodies diluted in the same buffer. Following intensive washing with $0.1 \%$ Tween-PBS, horse-radish peroxidase (HRP)-conjugated $\alpha$ mouse, $\alpha$-rabbit (Advansta) or $\alpha$-rat IgG (Santa Cruz Biotechnology) was used to detect primary antibodies bound to the membrane. Excess of secondary antibodies was rinsed away with $0.1 \%$ Tween-PBS and target proteins were indirectly visualized with Westernbright ECL Kit (Advansta) based on the enhanced chemiluminescence method. For immunoprecipitation and subsequent mass spectrometric analysis of recombinant PXDN oligomers, stable HEK293-PXDN-V5 cell lysate was co-incubated with Anti-V5 Agarose Affinity Gel (SigmaAldrich) in the presence of $1 \mathrm{mM}$ NEM. The beads were thoroughly washed with RIPA buffer and bound proteins were eluted with $1 \mu \mathrm{g} / \mathrm{ml} \mathrm{V5-His} \mathrm{peptide} \mathrm{(Caslo).} \mathrm{After} \mathrm{addition} \mathrm{of} \mathrm{non-}$ reducing Laemmli buffer, precipitated proteins were run on $4 \%$ polyacrylamide-SDS gels which were stained with Coomassie Blue Staining Solution (0.1\% Brillant Blue G (SigmaAldrich), 25\% methanol, 5\% acetic acid) or Acqua Stain Protein Staining Reagent (Acqua Science Ltd.). Protein bands giving intense signals were excised and further analyzed with mass spectrometry. Endogenously expressed PXDN was purified from HUVEC cells lysates (1\% Triton X-100, $50 \mathrm{mM} \mathrm{NaF}, 1 \mathrm{mM} \mathrm{NaVO}, 1 \mathrm{mM}$ PMSF, $10 \mu \mathrm{g} / \mathrm{ml}$ aprotinin, cOmplete Mini EDTA-Free Protease Inhibitor Cocktail Tablets, $140 \mathrm{mM} \mathrm{NaCl}, 30 \mathrm{mM}$ Tris pH 7.4) (data not shown). PXDN-containing complexes were precipitated with $\alpha$-hsPXDN antibodies 
bound to Protein G Agarose (Invitrogen, Life Technologies). After repeated washing (0.1\% Triton X-100, $1 \mathrm{mM}$ PMSF, $140 \mathrm{mM} \mathrm{NaCl}$ and $30 \mathrm{mM}$ Tris $\mathrm{pH}$ 7.4), purified proteins were eluted with non-reducing Laemmli buffer and then were run on $4 \%$ polyacrylamide-SDS gels. Stained protein bands were cut out and were submitted to mass spectrometric examination.

\section{Mass spectrometric analysis}

Excised protein bands of HUVEC or V5-positive precipitates were in gel digested with sidechain modified porcine trypsin (Promega) according to the protocol described at http://msf.ucsf.edu/ingel.html. The tryptic digests were analyzed on an LCQ-Fleet 3D Ion Trap or an LTQ-Orbitrap Elite Mass Spectrometer (Thermo Scientific) on-line coupled to a nanoACQUITY UPLC system (Waters). The tryptic peptides were loaded onto a trap column (Symmetry, C18 $5 \mu \mathrm{m}, 180 \mu \mathrm{m} \times 20 \mathrm{~mm}$ ) in 3\% solvent B at a flow rate of $10 \mu 1 / \mathrm{min}$, then separated on an ACQUITY UPLC BEH C18 column $(1.7 \mu \mathrm{m}, 75 \mu \mathrm{m} \times 200 \mathrm{~mm})$ at a flow rate of $450 \mathrm{nl} / \mathrm{min}$ using a gradient of $3-40 \%$ solvent B in 37 minutes. Solvent A was $0.1 \%$ formic acid in water; solvent B was $0.1 \%$ formic acid in acetonitrile. Data acquisition was performed in a data-dependent fashion. The most abundant multiply charged ions were selected for MS/MS. In the LCQ-Fleet 3D Ion Trap zoom scans were used to determine the charge state. In the LTQ-Orbitrap the surveys were measured in the Orbitrap followed by low resolution CID scans measured in the linear trap. The 3D ion trap data were converted to Mascot generic format using Mascot Distiller (version 2.1.1.0). LTQ-Orbitrap data were processed with PAVA (version 2010.09.30). Database search was performed against human entries in the 2011.06.10 NCBI non-redundant protein database (182800/14324397 sequences) using an inhouse Mascot server (version 2.2.04). For the 3D ion trap data monoisotopic masses with precursor mass tolerance of \pm 0.6 Da and fragment mass tolerance of \pm 1 Da were submitted, for the LTQ-Orbitrap data 10 ppm was set for the precursors and 0.6 Da for the fragments. 
Two missed cleavages were permitted. Carbamidomethylation of cysteine residues was set as fixed modification, and acetylation of protein N-termini, methionine oxidation and pyroglutamic acid formation from peptide $\mathrm{N}$-terminal glutamine residues as variable modifications. Peptide matches with $\mathrm{P}<0.05$ were accepted.

\section{Immunofluorescent labeling and laser confocal microscopy}

Cos7 cells and PXDN knockout MEFs expressing different recombinant PXDN constructs were grown on coverslips for 48 hours after transfection. After being washed in PBS, the cells were fixed in 4\% paraformaldehyde (PFA; Sigma-Aldrich)-PBS solution for 20 minutes. PFA was washed away with PBS and its remnants were quenched with $100 \mathrm{mM}$ glycine (Affymetrix)-PBS. The cells were then permeabilized for 20 minutes with $0.1 \%$ Triton X-100 in PBS supplemented with $1 \%$ BSA, while unpermeabilized samples were kept intact in $1 \%$ BSA-PBS. Coverslips were blocked in 3\% BSA-PBS and then were incubated with $\alpha-$ hsPXDN antibody diluted in 1\% BSA-PBS for 1 hour. After intensive washing Alexa Fluor 488 or 568 Goat Anti-Rabbit IgG (Life Technologies) diluted in 1\% BSA-PBS was added to the samples and the cells were incubated with the solution for another hour. Following the final washing coverslips were mounted with Mowiol 4-88 antifade reagent prepared from polyvinyl alcohol (Sigma-Aldrich), glycerol, $\mathrm{H}_{2} \mathrm{O}$ and Tris $\mathrm{pH}$ 8.5. Immunostaining of the same samples was also performed using $\alpha$-V5 as primary and Alexa Fluor 488 or 568 Goat Anti-Mouse IgG (Life Technologies) as secondary antibodies (data not shown).

Immunostained samples were analyzed with LSM710 confocal laser-scanning microscope (Carl Zeiss) with a 63x 1.4 numerical aperture plan Apochromat objective (Carl Zeiss). Final images were developed using "Maximum Intensity Projection" (MIP) postaquisition tool on z-stack images acquired from optical slices of $0.5 \mu \mathrm{m}$ thickness, covering the entire depth of the cells. 


\section{Amplex Red Peroxidase Assay}

Cos7 cells expressing different recombinant PXDN constructs were washed with PBS and lysed in $1 \%$ hexadecyltrimethylammonium bromide (CTAB; Sigma-Aldrich)-PBS solution 24 hours after transfection. Peroxidase activity of total cell lysates was measured and compared according to the instructions of Amplex Red Peroxidase Assay Kit (Molecular Probes, Life Technologies). Equal volume of cell lysate and Amplex Red Working Solution (Amplex Red Reaction Buffer containing $100 \mu \mathrm{M}$ Amplex Red Reagent and $2 \mathrm{mM} \mathrm{H}_{2} \mathrm{O}_{2}$ ) were mixed and fluorescence of the end-product resorufin was measured at $590 \mathrm{~nm}$ after a 30 minute-long incubation period at room temperature.

\section{Alignment of human heme peroxidase sequences}

Multiple sequence alignment of human myeloperoxidase (UniProt accession number: P05164), eosinophil peroxidase (UP Acc\#: P11678), lactoperoxidase (UP Acc\#: P22079), thyroid peroxidase (UP Acc\#: P07202) and peroxidasin (UP Acc\#: Q92626) was carried out using web format of Clustal-Omega program (15).

\section{Statistical analysis}

Western blots and immunofluorescence pictures demonstrated in this work are representative of at least three independent experiments with identical results. The exact number of separate experiments supporting the presented data is indicated in each figure legend. Data collected in Amplex Red Peroxidase Assays are presented as the means of resorufin fluoresence \pm standard error of mean (s.e.m.) of the number of experiments indicated $(n=6)$. 


\section{RESULTS}

\section{Collagen IV crosslinking is absent in PXDN-deficient mouse embryonic fibroblasts}

Collagen IV was recently identified as a substrate of PXDN (11). In a reaction thought to be universal in the animal kingdom the enzyme crosslinks the NC1 domains of collagen IV protomers through the formation of sulfilimine linkages. First we wanted to examine whether crosslinking collagen IV molecules is a physiological function of the endogenously expressed mammalian PXDN. To achieve this goal we prepared mouse embryonic fibroblasts (MEFs) from PXDN-deficient animals which were created by the zinc-finger endonuclease technique. To confirm the knockout phenotype we studied the presence of the PXDN protein in lysates of wild-type and PXDN knockout cells. Figure 1A (left panel) shows that PXDN was absent in MEFs prepared from PXDN mutant animals. We digested wild-type and knockout MEF lysates with collagenase and analyzed the ratio between NC1 dimers and monomers by Western blot. While digests of wild-type MEFs contained both dimers and monomers, crosslinked NC1 domains were totally absent in knockout lysates (Figure 1B). These experiments proved the essential role of mammalian PXDN in collagen IV crosslinking.

\section{Trimerization of heterologously- and endogenously expressed mammalian PXDN}

In our previous study we demonstrated the widespread expression of the $p x d n$ gene and the presence of the protein in different types of human primary cells including pulmonary, dermal fibroblasts and HUVECs (9). To get a better understanding of the function of this complex protein first we decided to study the supramolecular organization of PXDN which was previously found to oligomerize into trimers in Drosophila (6). When PXDN was detected in non-reduced samples of HUVECs, three bands were recognized at approximately 160, 300 and over $500 \mathrm{kD}$, respectively (Figure 2A, left panel). Importantly, the band with the highest 
molecular mass was the most abundant detected, suggesting that PXDN could form oligomers or interact with other unknown proteins. We created a HEK293-based cell line that stably expressed high amount of a V5 epitope-tagged version of PXDN. When lysates from this cell line were analyzed under non-reducing conditions, the same pattern of PXDN positive bands was observed (Figure 2A, left panel). In accordance with previous reports (8;9), in reduced samples PXDN was detected at the expected size of $165 \mathrm{kD}$ (Figure 2A, right panel). We purified the V5 epitope-tagged PXDN protein from transfected HEK293 cells by immuneaffinity chromatography. After Coomassie blue staining of the separated proteins we detected three bands at the molecular masses already described above (Figure 2B). Mass spectrometric analysis revealed that the bands contained only PXDN (Supplementary figure 2). In other experiments we used PXDN-specific antibody and immunoprecipitated the protein from HUVEC lysates and analyzed the isolated proteins by mass spectrometry. These experiments confirmed that the band at the $500 \mathrm{kD}$ marker contained only peroxidasin (Supplementary figure 3). Altogether, the above described experiments suggest that mammalian PXDN forms oligomers and based on its electrophoretic mobility, the dominant form appears to be trimeric.

\section{Disulphide bond formation between conserved cysteines mediates the oligomerization of PXDN}

In subsequent experiments we investigated the mechanism of PXDN oligomerization. Dimerization of LPO was previously described to be dependent on the enzymatic activity of the protein (16), therefore we examined if the peroxidase activity of PXDN is required for the assembly of PXDN trimers. We mutated two highly-conserved amino acids which are located in the active site of the enzyme and were previously proved to be essential for enzymatic activity (13). As shown in Figure 3A, the Q823W, D826E mutations erased the peroxidase activity of PXDN while oligomerization of the protein remained unaffected (Figure 3B). 
Dimerization through cysteine residues has been described for other members of the peroxidase family, including MPO (13). We aimed to locate the positions of cysteines that mediate coupling of PXDN monomers. To achieve this, first we expressed truncated versions of PXDN and studied the oligomerization of the mutant forms. The N-terminally truncated mutant, PXDN ${ }_{1-26+696-1479}$, contained the signal peptide of the protein but lacked the leucinerich repeats and C2-type immunoglobulin domains which represent the $\mathrm{N}$-terminal half of the protein. Another mutant $\left(\mathrm{PXDN}_{1-1412}\right)$ lacked the C-terminal vWF C-type domain which contains ten cysteine residues, while the third construct $\left(\mathrm{PXDN}_{1-26+696-1412}\right)$ contained the signal sequence but all three regions mentioned above were missing. Importantly, none of the described modifications affected the ability of PXDN to oligomerize (Figure 4A). These experiments indicated that cysteine residues which mediate the coupling of PXDN monomers are located between amino acids 696 and 1412.

Earlier structural studies including crystallographic analysis of MPO and LPO identified highly conserved cysteines that stabilize the proteins' structure through the formation of intramolecular disulfide bonds $(17 ; 18)$. Alignment of PXDN with protein sequences of other mammalian heme peroxidases including MPO and LPO helped us to locate cysteines between the 696 and 1412 positions which are likely involved in intramolecular disulfide bridge formation in PXDN as well (Supplementary figure 4). Besides those 14 residues we identified 4 additional cysteines at positions 736, 1315, 1316 and 1319, respectively (Supplementary figure 4). We created several mutant constructs to test the possible role of individual cysteines in intermolecular disulphide bridge formation. First we studied the oligomerization of C1315S, C1316S and C1319S single mutants, respectively. Disappearance of the highest molecular mass form of PXDN was only observed when C1315 was mutated (Figure 4B). We also tested different combinations of the C-terminal mutations and these experiments confirmed the importance of C1315 in the oligomerization process 
(Figure 4B). When all three cysteines were mutated (C1315S, C1316S, C1319S), only the monomeric form was observed. Interestingly, in the case of the C1315S and C1319S mutants we observed additional bands above the expected molecular mass of the oligomeric forms. It is possible that disufide formation between a hitherto unknown chaperone and PXDN is responsible for the appearance of these bands which were not analyzed further. Our next target, the $\mathrm{C} 736$ residue is also conserved among PXDNs of different species examined (data not shown). Figure 4C shows that the C736S mutant did not form the high molecular mass oligomer and only dimeric and monomeric forms were detected. Importantly, simultaneous mutations of C736 and C1315 resulted in the exclusive formation of PXDN monomers, thus proving the key role of C736 and C1315 in the oligomerization process (Figure 4C).

\section{Trimerization of PXDN is essential for optimal collagen IV crosslinking}

PXDN is a secreted peroxidase with adhesive properties so we decided to test the structural basis for each of its previously described features. To investigate the structural requirements for secretion we analyzed the cell culture medium of transfected Cos7 cells for its PXDN content by Western blot. To exclude the possibility that the PXDN signal origins from unattached cells, we also tested the medium for actin. As shown in Figure 5, the presence of the secretory signal (between amino acids 1 and 26) was necessary and sufficient to transport the wild-type protein as well as different mutant PXDN forms to the extracellular space.

Next we examined whether the enzymatic activity of PXDN is dependent on its oligomeric structure and presence of protein modules that lay outside of the peroxidase domain. When tested in Amplex Red Peroxidase Assay, the C736S, C1315S double mutant and the protein lacking the C-terminal vWF domain were equally active as the wild-type enzyme. On the other hand, the concurrent absence of $\mathrm{N}$-terminal leucine-rich repeats and IgG-like domains erased the catalytic activity of PXDN (Figure 6A). Results of the 
previously described experiments on $\mathrm{KO}$ MEFs suggested that these cells offer a promising tool for studying the crosslinking activity of different PXDN mutants in rescue experiments. We expressed wild-type PXDN and different mutant forms of the protein in knockout MEFs (Figure 6B) and studied the formation of NC1 dimers by Western blot analysis. As expected, wild-type PXDN efficiently catalyzed the formation of NC1 dimers, whereas the loss of function Q823W, D826E mutant did not support the formation of crosslinks (Figure 6C). The PXDN construct lacking the C-terminal vWF C-type domain ( $\left.\mathrm{PXDN}_{1-1412}\right)$ was equally active as the wild-type protein, but the monomeric C736S, C1315S form was less effective, although it retained its ability to support the crosslinking reaction (Figure 6C). Since the wild-type protein and the monomeric mutant were equally active in the Amplex Red assay, we were interested in finding the explanation for their different activity in collagen crosslinking experiments.

When we immunostained PXDN in Cos7 cells that heterologously expressed the protein we observed a prominent localization within the endoplasmic reticulum (ER) (Figure 7) as described in our earlier paper (9). Besides the ER localization (Figure 7A-D) we also observed punctuate structures that were strongly positive for PXDN (Figure 7A, B, D). When we stained unpermeabilized cells, PXDN was detected only at these structures indicating their localization on the surface of the cells. The absence of the peroxidase activity or the lack of the C-terminal vWF C-type domain did not alter punctuate staining pattern of PXDN (Figure 7E, F, I), however, the monomeric form failed to appear on the surface of the cells (Figure 7G, H). In subsequent experiments we compared the localization of the wild-type protein with that of the monomeric C736S, C1315S mutant in PXDN-deficient MEFs. The wild-type protein again was observed in the punctuate pattern, which was also detectable at the plasma membrane of unpermeabilized cells (Figure 8A, C). The monomeric form, however, was accessible to the antibody only after permeabilization of the cells (Figure 8B, D, E). 
Altogether, these experiments suggested that attachment of secreted PXDN to the cellular surface requires the formation of trimers. 


\section{DISCUSSION}

Collagen IV is a major component of basement membranes that are thin extracellular matrix sheets underlying endothelial and epithelial cells and encapsulating muscle, fat and Schwann cells (19). Although the role of collagen IV in providing mechanical support for cells has been known for a while, regulatory functions of the protein are also increasingly being recognized in development and tissue repair (20;21). Assembly of the collagen IV network is a complex process, where crosslinking of collagen IV protomers leads to the formation of a scaffold that interacts with other basement membrane proteins. A recently identified mechanism of collagen IV crosslinking is the formation of sulfilimine links between interfacing NC1 domains, a coupling mechanism which has been identified only in collagen IV so far (12). The animal heme peroxidase PXDN has been shown to catalyze the formation of sulfilimine links in a reaction, where the enzyme produces hypohalous acids as intermediates for the oxidation of a highly conserved Met residue in the NC1 domain (11). Although peroxidase inhibitors were previously shown to inhibit collagen IV crosslinking in a murine embryonic carcinoma cell line (11), evidence for PXDN-mediated coupling of NC1 domains being a physiological pathway of ECM formation in mammals has been lacking so far. In our experiments we compared the formation of NC1 dimers between wild-type and PXDNdeficient MEFs and found that KO cells failed to form crosslinked NC1 domains. These experiments also indicated that compensatory pathways for sulfilimine-mediated collagen IV coupling unlikely exist in mammals, highlighting the physiological significance of the PXDNcatalyzed reaction. We did not observe any difference between the collagen IV content of wild-type and PXDN KO MEFs (data not shown), suggesting that the absence of sulfinimine links does not lead to a compensatory increase of collagen IV synthesis. 
Several molecular details are known about the organization of basement membranes, including the molecular architecture and interactions of proteins that orchestrate the assembly process (19). On the other hand, no structure-function analysis on PXDN has so far been reported and little is known about the supramolecular organization of the protein. Drosophila PXDN was originally described to form trimers, but the mechanism of oligomerization was not pursued further by the authors (6). Our experiments on both endogenously- and heterologously-expressed PXDN revealed that the mammalian protein also exists in a trimeric form. PXDN is unique hybrid protein composed of domains that are characteristic of constituents of the ECM and a peroxidase domain that is highly homologous to other members of the animal heme peroxidase family (7). Interestingly, multimerization is a shared feature of both heme peroxidases and non-enzymatic BM proteins including laminin and collagen IV itself. LPO, for example, was described to form dimers held together by dityrosine links, the formation of which is dependent on the peroxidase activity of the enzyme (16). A distinct heme peroxidase, MPO also exists in dimers, however its structure is stabilized by intermolecular disulphide bridges (13). For PXDN we found the peroxidase activity dispensable for supramolecular assembly and intermolecular disulfide bridge formation was identified as the key mechanism of oligomerization. Creating a series of truncation and point mutants enabled us to identify two cysteines (C736 and C1315) that are responsible for the assembly of the mammalian PXDN trimer. When these cysteines were mutated simultaneously, only monomeric PXDN was detected. Multiple sequence alignment of PXDN sequences from different species revealed that these cysteines are highly conserved across the animal kingdom, suggesting a universal mechanism for PXDN oligomerization. Furthermore, we recently identified a novel homolog of PXDN, PXDNL (3), which also contains these cysteines at identical positions. Sequence similarity between the homologs might be indicative for PXDNL oligomerization as well. There are two additional cysteines 
(C1316 and C1319) in the proximity of C1315. Point mutations of these residues, either alone or in combination, did not interfere with the oligomerization process. When C1315 was mutated into serine, while C1316 and/or C1319 were left intact, we observed dimers as dominant forms of PXDN. We propose that in the absence of $\mathrm{C} 1315$ the remaining cysteines can still form "forced" intermolecular disulfide bridges, resulting the assembly of dimers. This explanation is supported by the observation that the triple mutant (C1315S, C1316S, C1319S) form of PXDN is exclusively monomeric.

Close to its C-terminus, the Drosophila PXDN contains an amphipatic $\alpha$-helical region, which was proposed to form homotrimeric coiled coil (6). A homologous region between amino acids 1383 and 1411 is also recognizable in the mammalian protein and we assume that trimerization via this region positions the $\mathrm{C} 736$ and $\mathrm{C} 1315$ residues to form intermolecular disulfide bonds leading to the assembly of a stabilized PXDN trimer.

When tested in Amplex Red Peroxidase Assay, we found that the monomeric (C736S, C1315S) form was equally active as the wild-type protein. This result indicates that although the point mutations interfered with supramolecular organization of the protein, they did not affect the activity of the peroxidase domain on an artificial substrate. Exclusion of the very Cterminal vWF C-type domain did not affect the enzymatic activity either, but deletion of the $\mathrm{N}$-terminal half of the protein including the leucine-rich repeats and the immunoglobulin $\mathrm{C} 2$ domains erased the peroxidase activity of PXDN. We assume that this latter modification interferes with folding of the peroxidase domain by an unknown mechanism, which was not pursued further in this study. To investigate the biological activity of the different PXDN mutants in a more physiological setting, we reintroduced them into PXDN-deficient MEFs and examined whether they were able to rescue the collagen IV crosslinking defect of the knockout cells. Interestingly, when compared to the activity of the wild type protein, we found the monomeric (C736S, C1315S) form less effective in these experiments, suggesting 
that optimal coupling of $\mathrm{NC} 1$ domains requires trimerization of the protein. On the other hand, the lack of the vWF C-type domain did not affect the crosslinking activity of PXDN, which was absolutely dependent on the peroxidase activity of the protein.

When we studied the cellular localization of the different PXDN mutants we observed interesting differences, which might explain the previously discussed discrepancies in collagen IV crosslinking. All of the examined constructs showed the characteristic appearance in the endoplasmic reticulum, which was previously described by us for both endogenouslyand heterologously expressed PXDN (9). Intriguingly, wild-type PXDN or mutants which were previously shown to retain their ability to form trimers, were also detected in separate, punctuate-like structures showing intense PXDN labeling. These PXDN-positive structures were closely associated with the cells but they were also accessible for immunostaining in non-permeabilized cells, indicative of an extracellular localization. Importantly, the monomeric PXDN mutant did not show this staining pattern, indicating that trimer formation is a prerequisite for this unique localization. This result was particularly interesting in the light of the fact that the monomeric form was also secreted from the cells. Crosslinking of collagen IV protomers through sulfilimine bonds is an extracellular event (20), however the localization of the reaction is currently unknown. We assume that the previously unknown PXDN-positive "hot spots" on the cell surface might represent sites of collagen IV crosslinking, explaining the results of experiments where the multimeric form of PXDN showed maximal crosslinking activity. Similar to other heme peroxidases, PXDN's enzymatic activity is also dependent on the availability of hydrogen peroxide, which is supplied to the enzyme by a currently unknown cellular source. Crosslinking collagen IV on the cell surface may be advantageous by reducing the distance between the coupling reaction and the sites of $\mathrm{H}_{2} \mathrm{O}_{2}$ release. On the other hand, independent of peroxidase chemistry the membrane-attached PXDN-positive structures may have an important role in adhesion to other components of the 
BM through leucine-rich repeats, immunoglobulin C2 and vWF C-type motifs that lay outside of the peroxidase domain and are frequently found in other adhesion proteins $(22 ; 23)$. In this regard it is particularly interesting that PXDNL, which is highly similar to PXDN, has lost its enzymatic activity during evolution (3). However, in spite of this molecular "defect" PXDNL probably has important, presumably adhesive function, suggested by its enrichment at the intercalated disks in the cardiac tissue. Future experiments on the interactions of these modules will be essential for understanding the role of PXDN in the synthesis and function of basement membranes.

\section{ACKNOWLEDGEMENTS}

We are grateful to Beáta Molnár and Katalin Meczker for technical assistance.

This work was supported by grants from the Hungarian Research Fund (OTKA K106138),

National Office for Research and Technology Ányos Jedlik Programme AVINOMID (OM00087/2007) and by BAROSS-DA07-DA-ESZK-07-2008-0036 grant (to the Biological Research Centre, Hungarian Academy of Sciences). Miklós Geiszt is supported by "Momentum" grant from the Hungarian Academy of Sciences.

\section{CONFLICT OF INTEREST}

The authors declare no competing financial interests. 


\section{REFERENCES}

1. Winterbourn, C. C. (2013) Methods Enzymol. 528, 3-25

2. Davies, M. J., Hawkins, C. L., Pattison, D. I., and Rees, M. D. (2008) Antioxid.Redox.Signal. 10, 1199-1234

3. Peterfi, Z., Toth, Z. E., Kovacs, H. A., Lazar, E., Sum, A., Donko, A., Sirokmany, G., Shah, A. M., and Geiszt, M. (2014) Cardiovasc.Res. 101, 393-399

4. Heinecke, J. W. and Shapiro, B. M. (1992) J.Biol.Chem. 267, 7959-7962

5. Edens, W. A., Sharling, L., Cheng, G., Shapira, R., Kinkade, J. M., Lee, T., Edens, H. A., Tang, X., Sullards, C., Flaherty, D. B., Benian, G. M., and Lambeth, J. D. (2001) J.Cell Biol. 154, 879-891

6. Nelson, R. E., Fessler, L. I., Takagi, Y., Blumberg, B., Keene, D. R., Olson, P. F., Parker, C. G., and Fessler, J. H. (1994) EMBO J. 13, 3438-3447

7. Peterfi, Z. and Geiszt, M. (2014) Trends Biochem.Sci. 39, 305-307

8. Cheng, G., Salerno, J. C., Cao, Z., Pagano, P. J., and Lambeth, J. D. (2008) Free Radic.Biol.Med. 45, 1682-1694

9. Peterfi, Z., Donko, A., Orient, A., Sum, A., Prokai, A., Molnar, B., Vereb, Z., Rajnavolgyi, E., Kovacs, K. J., Muller, V., Szabo, A. J., and Geiszt, M. (2009) Am.J.Pathol. 175, 725-735

10. Soudi, M., Zamocky, M., Jakopitsch, C., Furtmuller, P. G., and Obinger, C. (2012) Chem.Biodivers. 9, 1776-1793

11. Bhave, G., Cummings, C. F., Vanacore, R. M., Kumagai-Cresse, C., Ero-Tolliver, I. A., Rafi, M., Kang, J. S., Pedchenko, V., Fessler, L. I., Fessler, J. H., and Hudson, B. G. (2012) Nat.Chem.Biol. 8, 784-790

12. Vanacore, R., Ham, A. J., Voehler, M., Sanders, C. R., Conrads, T. P., Veenstra, T. D., Sharpless, K. B., Dawson, P. E., and Hudson, B. G. (2009) Science 325, 1230-1234

13. Furtmuller, P. G., Zederbauer, M., Jantschko, W., Helm, J., Bogner, M., Jakopitsch, C., and Obinger, C. (2006) Arch.Biochem.Biophys. 445, 199-213

14. Horikoshi, N., Cong, J., Kley, N., and Shenk, T. (1999) Biochem.Biophys.Res.Commun. 261, 864-869

15. Sievers, F., Wilm, A., Dineen, D., Gibson, T. J., Karplus, K., Li, W., Lopez, R., McWilliam, H., Remmert, M., Soding, J., Thompson, J. D., and Higgins, D. G. (2011) Mol.Syst.Biol. 7, 539

16. Lardinois, O. M., Medzihradszky, K. F., and Ortiz de Montellano, P. R. (1999) J.Biol.Chem. 274, 35441-35448 
17. Fiedler, T. J., Davey, C. A., and Fenna, R. E. (2000) J.Biol.Chem. 275, 11964-11971

18. Singh, A. K., Singh, N., Sharma, S., Shin, K., Takase, M., Kaur, P., Srinivasan, A., and Singh, T. P. (2009) Biophys.J. 96, 646-654

19. Yurchenco, P. D. (2011) Cold Spring Harb.Perspect.Biol. 3,

20. Khoshnoodi, J., Pedchenko, V., and Hudson, B. G. (2008) Microsc.Res.Tech. 71, 357-370

21. Ortega, N. and Werb, Z. (2002) J.Cell Sci. 115, 4201-4214

22. Kobe, B. and Kajava, A. V. (2001) Curr.Opin.Struct.Biol. 11, 725-732

23. Walsh, F. S. and Doherty, P. (1997) Annu.Rev.Cell Dev.Biol. 13, 425-456 


\section{FIGURE LEGENDS}

\section{Figure 1. Mammalian PXDN is essential for collagen IV protomer crosslinking.}

A Western blot analysis of mouse embryonic fibroblast (MEF) lysates (TCL) prepared from wild-type (WT) and PXDN knockout (KO) animals confirmed the absence of PXDN in the mutant cells.

B Following collagenase digestion of cell lysates (CDL) crosslinked dimeric and uncrosslinked monomeric NC1 domains of collagen IV were separated by SDS-PAGE. When tested for the $\alpha 2$ isoform of collagen IV, both $\mathrm{NC} 1$ dimers and monomers could be detected in wild-type samples whereas in PXDN-deficient digests only the uncrosslinked form was present.

Representative blots of three separate experiments with identical results are shown in both panels.

\section{Figure 2. Western blot analysis of PXDN-containing protein complexes.}

A Total cell lysates of HUVEC and stably transfected HEK293-PXDN-V5 cells were tested for PXDN expression under reducing or non-reducing conditions by SDS-PAGE. According to its calculated molecular mass reduced PXDN appeared at $165 \mathrm{kD}$ whereas in non-reduced samples the most intense signal was detected at $500 \mathrm{kD}$ along with two weaker bands at 300 $\mathrm{kD}$ and $160 \mathrm{kD}$. We observed the same band pattern in five independent immunoblots.

B Coomassie-staining of immunopurified PXDN-V5 revealed a similar pattern of molecular masses under non-reducing conditions in three separate experiments. Mass spectrometric analysis identified PXDN as the only component of the excised bands, which - along with their apparent molecular masses - suggests the three bands to be trimeric, dimeric and monomeric forms of PXDN. 
Figure 3. Relationship between catalytic activity and oligomer formation of PXDN.

A Cos7 cells transfected with wild-type (WT) or Q823W, D826E double mutant PXDN constructs were lysed and their peroxidase activity was measured by Amplex Red Peroxidase Assay. Cells expressing the mutant protein lacking conserved amino acids essential for heme binding showed low peroxidase activity equal to mock-transfected cells whereas increased resorufin fluorescence was measured in cells expressing the wild-type construct (representative measurement).

B The potential for oligomerization of the same constructs was investigated by Western blot analysis of transiently transfected HEK293 cell lysates. The loss of peroxidase activity of the Q823W, D826E mutant did not affect oligomerization indicating that enzymatic activity of PXDN is not essential for the process. Representative of six separate experiments with identical results is presented.

\section{Figure 4. Identification of protein domains and amino acids involved in PXDN}

\section{oligomerization.}

A N- and C-terminally truncated PXDN constructs were designed to study the role of each protein domain in the assembly of PXDN oligomers. Western blot analysis of transiently transfected HEK293 cell lysates showed that neither combined nor separate absence of von Willebrand factor type $\mathrm{C}$ domain, leucine-rich repeats or immunoglobulin $\mathrm{C} 2$ domains prevented oligomerization process as long as the original signal sequence was present at the $\mathrm{N}$-terminus of the recombinant construct. B, C To study their involvement in PXDN oligomer formation, unique cysteines located between positions 696 and 1412 were mutated to serines in various combinations and the constructs generated were expressed in HEK293 cells. Western blot demonstrated that the absence of C1316, C1319 either alone or in 
combination did not prevent oligomerization of PXDN, whereas constructs lacking C1315 or C736 were able to assemble into dimers but not into the dominant trimeric form.

Simultaneous mutation of Cys736 and Cys1315 or Cys1315, CysC1316 and Cys1319 entirely abolished oligomer formation and only monomeric PXDN could be detected.

Western blots on lysates from separately transfected cells were performed four (A) or six (B, C) times with the same result.

Figure 5. Identification of the sequence requirement for PXDN secretion.

Culture supernatant of Cos7 cells expressing mutant and $\mathrm{N}$ - or C-terminally truncated PXDN was collected and tested for recombinant protein content. Western blot of total cell lysates confirmed nearly equal transfection efficiency of the examined plasmids. All constructs containing the first 26 amino acids of PXDN were present in the cell culture medium whereas no V5 signal could be detected in the supernatant of PXDN $695-1479$-transfected cells. These data confirmed in four separate experiment suggest that presence of the original signal sequence is essential and sufficient for secretory production of PXDN.

\section{Figure 6. Characterisation of structural requirements for PXDN enzymatic activity.}

A Peroxidase activity of Cos7 cell lysates expressing different recombinant PXDN constructs was measured in Amplex Red Peroxidase Assay. Resorufin generation by wild-type PXDN (WT) was not affected by C736S, C1315S mutations or the absence of the von Willebrand factor type $\mathrm{C}$ domain $\left(\mathrm{PXDN}_{1-1412}\right)$ but was abolished in the $\mathrm{N}$-terminally truncated $\mathrm{PXDN}_{1-}$ 26+696-1479 construct and the Q823W, D826E double mutant ( $\mathrm{n}=6, \pm$ s.e.m.).

B To examine their ability to rescue collagen IV crosslinking function of the wild-type protein, certain constructs were expressed in PXDN-deficient mouse embryonic fibroblasts 
(MEF). Western blot of total cell lysates confirmed equal transfection efficiency and expression of PXDN proteins.

C Western blot demonstrating the appearance of NC1 dimers in collagenase digested MEF lysates (CDL) confirms that WT, PXDN ${ }_{1-1412}$ and C736S, C1315S constructs were also able to restore crosslinking of collagen IV $\alpha 2$ chains with the latter showing weaker activity. The Q823W, D826E double mutant could not restitute NC1 dimerization underlining that catalytic activity of PXDN is indispensable for collagen crosslinking.

The shown immunoblots are representative of three experiments with the same results.

\section{Figure 7. Investigation of structural determinants of PXDN subcellular localization.}

A-D Immunostaining of heterologously expressed recombinant PXDN constructs was carried out with permeabilization of transfected Cos7 cells. Beside dominant endoplasmic reticulum (ER) localization of all examined constructs, wild-type (A), Q823W, D826E (B) and PXDN 1 ${ }_{1412}$ (D) proteins were observed along the plasma membrane of permeabilized cells (marked with arrows).

E-I Immunostaining of heterologously expressed recombinant PXDN constructs was carried out without permeabilization of transfected Cos7 cells.Unlike the ER-associated staining, signal corresponding to the membrane-attached, punctuate structures were also detected in unpermeabilized samples supporting their presence on the extracellular surface of the cells $(\mathbf{E}$, F, I, marked with arrows). Although presence of intact cells was confirmed by fake colour LUT visualization in each observed field $(\mathbf{H})$, virtually no PXDN signal could be recognized in unpermebilized samples of C736S, C1315S-expressing Cos7 cells (G). The bar indicates $20 \mu \mathrm{m}$. Images were obtained from six identically transfected and immunostained samples.

Figure 8. Verification of dual PXDN localization in MEFs. 
A-B Permeabilized samples of PXDN knockout mouse embryonic fibroblasts (MEF) transfected with wild-type (WT) PXDN or C736S, C1315S mutant construct were immunostained for PXDN. Simultaneous endoplasmic reticulum and plasma membrane localization was observed only in cells expressing the wild-type construct (A) whereas the mutant form localized exclusively to the endoplasmic reticulum (B). The bar represents 20 $\mu \mathrm{m}$.

C-E Non-permeabilized samples of PXDN knockout mouse embryonic fibroblasts (MEF) transfected with wild-type (WT) PXDN or C736S, C1315S mutant construct were immunostained for PXDN. Without prior permeabilization only the membrane-bound, extracellular wild-type PXDN remained detectable (C) whereas C736S, C1315S mutant samples did not show any specific staining (D). (Fake colour LUT visualisation indicated intact cells in the same visual fields (E).) The bar represents $20 \mu \mathrm{m}$.

Transfection and immunostaining of the cells were repeated four separate times to confirm reproducibility of the data.

\section{Supplementary figure 1. Schematic representation of recombinant PXDN constructs}

\section{used in this study.}

Beside its catalytic domain PXDN contains several other regions characteristic for extracellular matrix proteins including a secretory signal sequence $(\mathrm{S})$, leucine-rich repeats (LRR), immunoglobulin-like C2 domains (Ig C2) and a von Willebrand factor C-type domain (vWF C). To carry out structure-function analysis of the protein we designed a number of truncated and mutated, V5 epitope-tagged recombinant constructs that were tested in both Western blot analyses and functional assays. Amino acid regions of PXDN constituting a particular truncated construct were indicated in the subscript of the construct's name according to their positions in the original sequence. Single, double and triple point mutant 
constructs labeled with the introduced amino acid substitutions and their positions were generated by site-directed mutagenesis of full-length PXDN.

Supplementary figure 2. Mass spectrometric identification of PXDN in the $500 \mathrm{kD}$ band immunopurified from transfected HEK293-PXDN-V5 cells. Tryptic digest of the protein band was analyzed by LC-MS/MS. Mascot search of the data identified PXDN as the sole constituent.

\section{Supplementary figure 3. Mass spectrometric analysis of $500 \mathrm{kD}$ protein band} immunoprecipitated from HUVEC cells. LC-MS/MS was used to analyse protein fragments following trypsin digestion of the band. PXDN was identified as its only component by Mascot search of the data.

\section{Supplementary figure 4. Identification of unique cysteine residues in the peroxidase} domain of PXDN.

Multiple sequence alignment indicates that human heme peroxidases share 14 highly conserved cysteine residues that have previously been reported to form intramolecular disulphide bonds stabilizing tertiary structures of promyeloperoxidase and lactoperoxidase. PXDN contains an additional cysteine at position 736 (in blue box) and other three residues in close proximity to the C-terminus of peroxidase domain at positions $1315,1316,1319$ (in pink boxes) that are candidates to form interchain linkages during the assembly of PXDN oligomers. 


\section{Figure 1}

$\mathbf{A}$

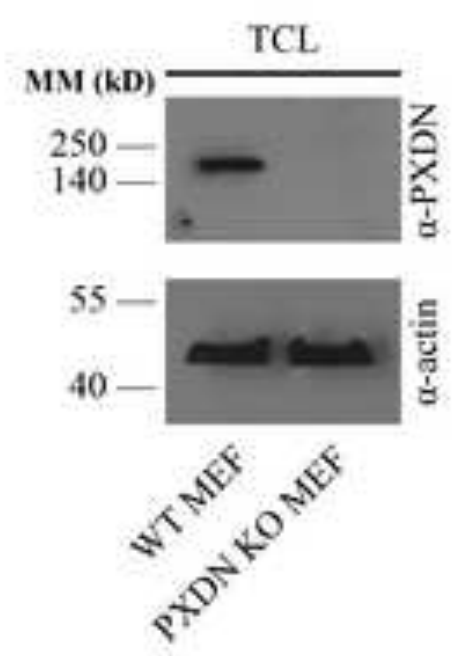

B

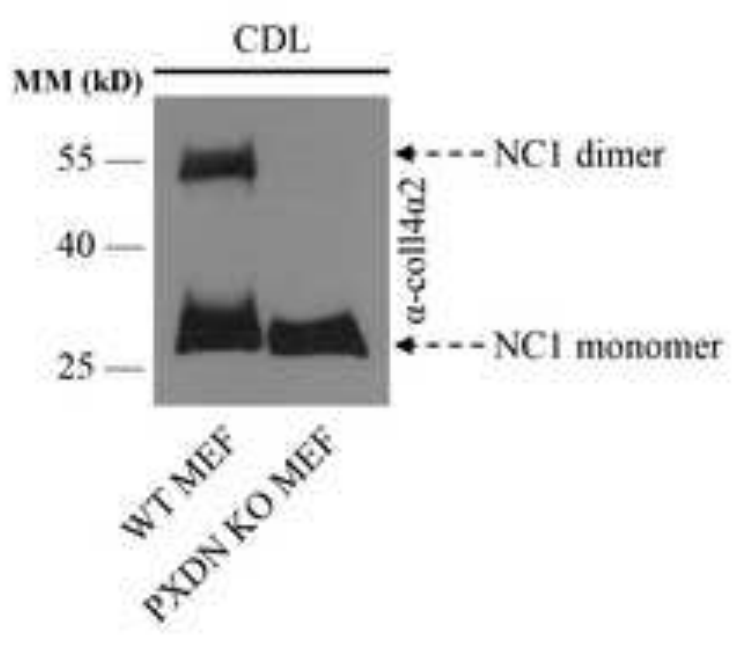


Figure 2

A

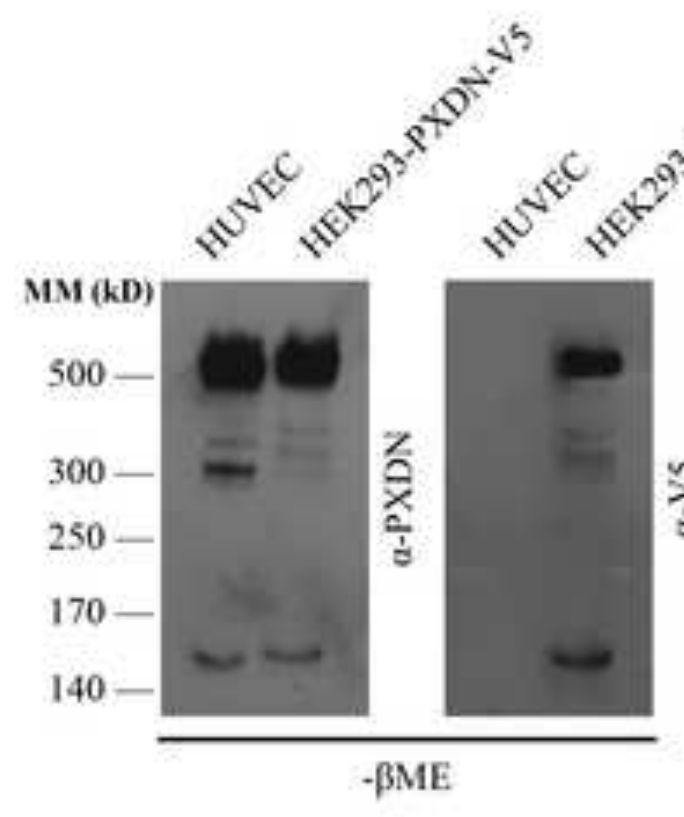

B

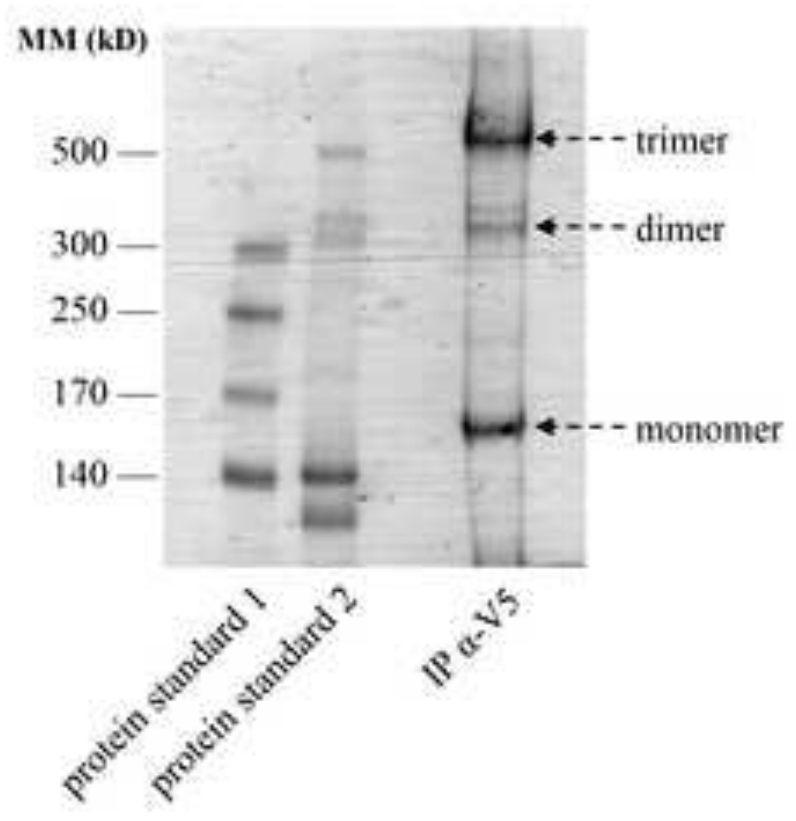


A

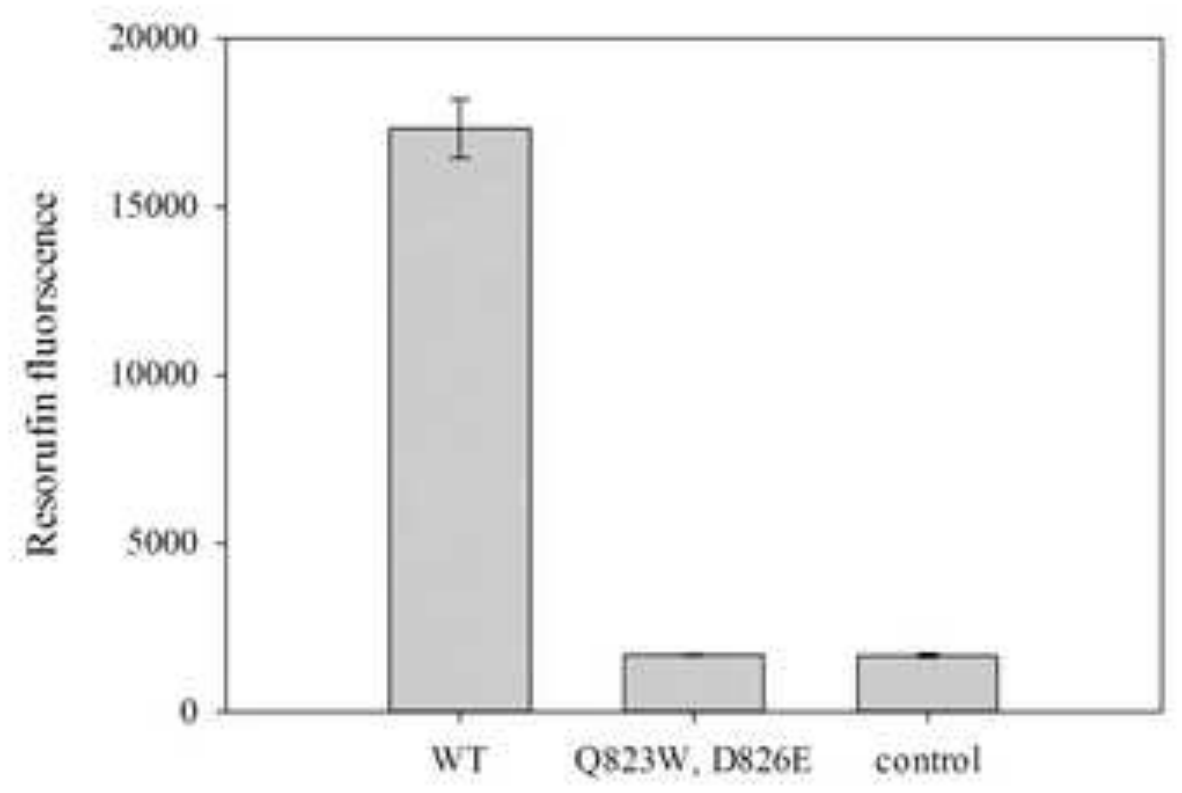

B

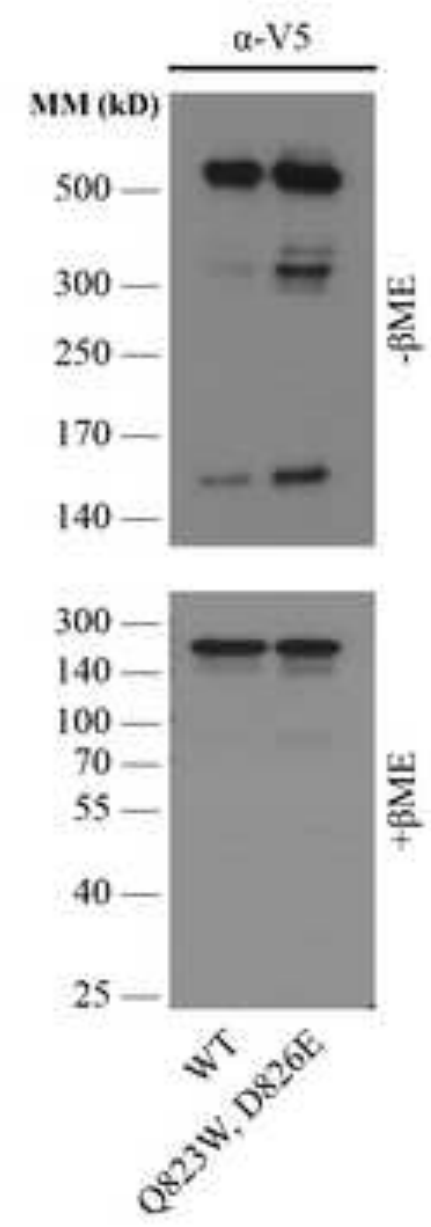

$\frac{1}{4}$

Figure 3.

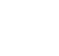


Figure 4

A

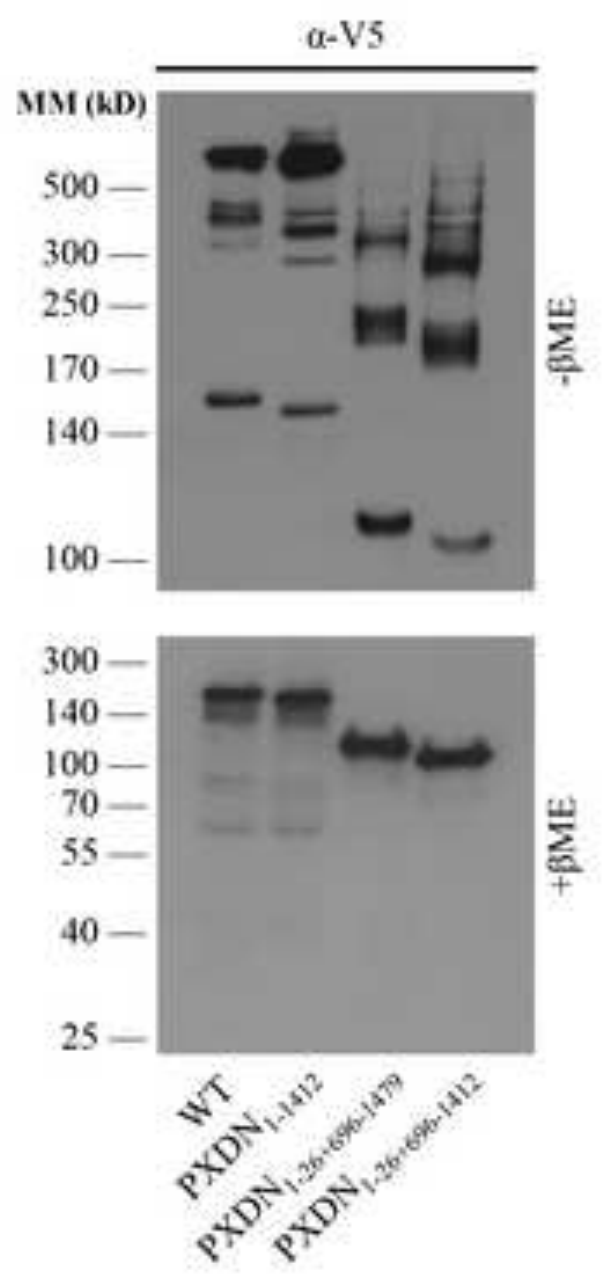

B
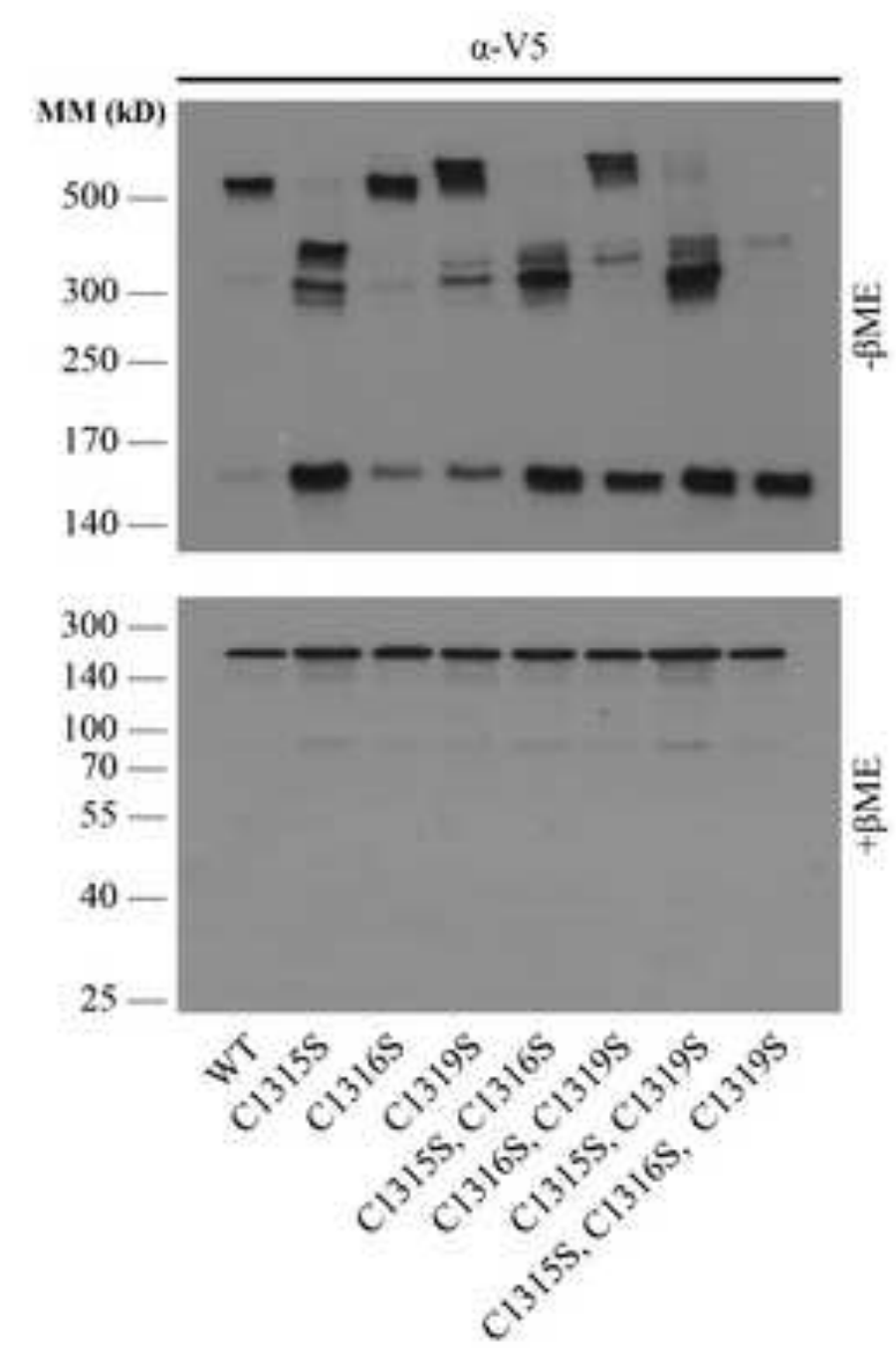

C

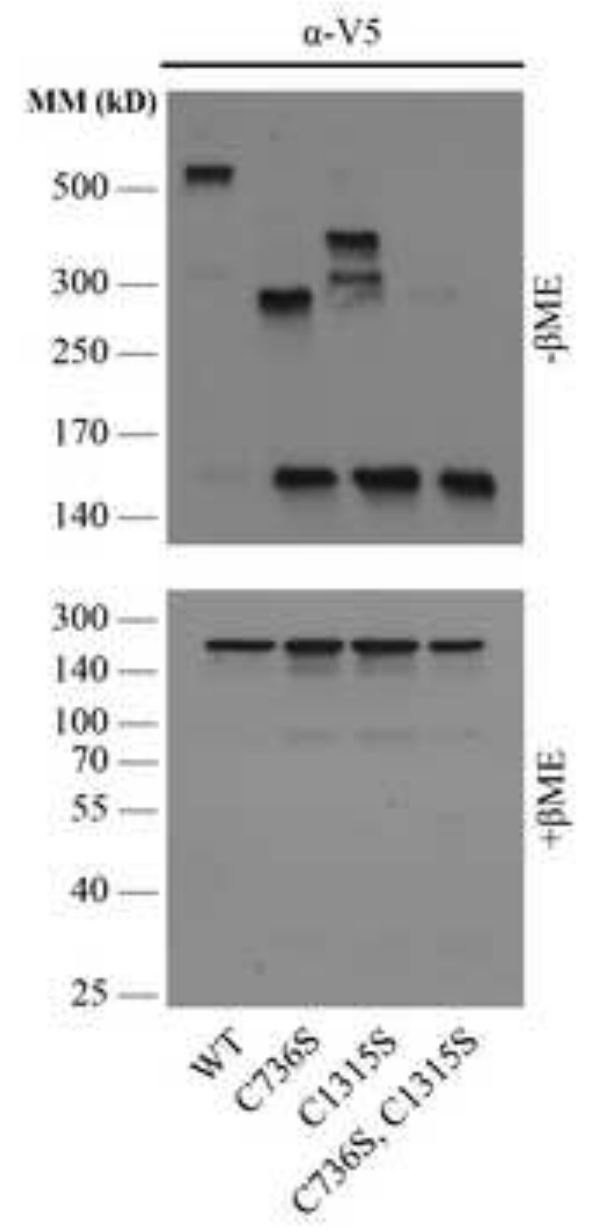


Click here to download high resolution image

Figure 5

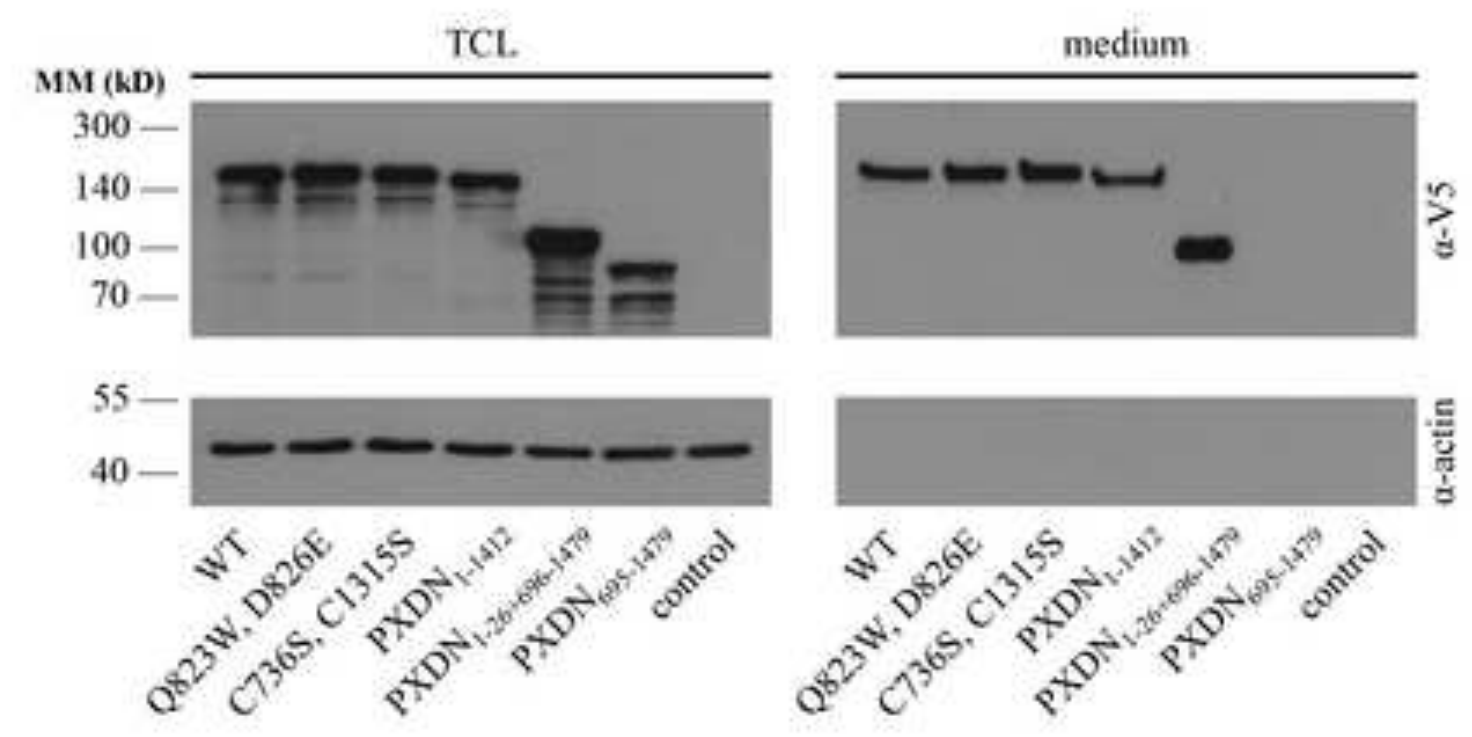


Figure 6.

A

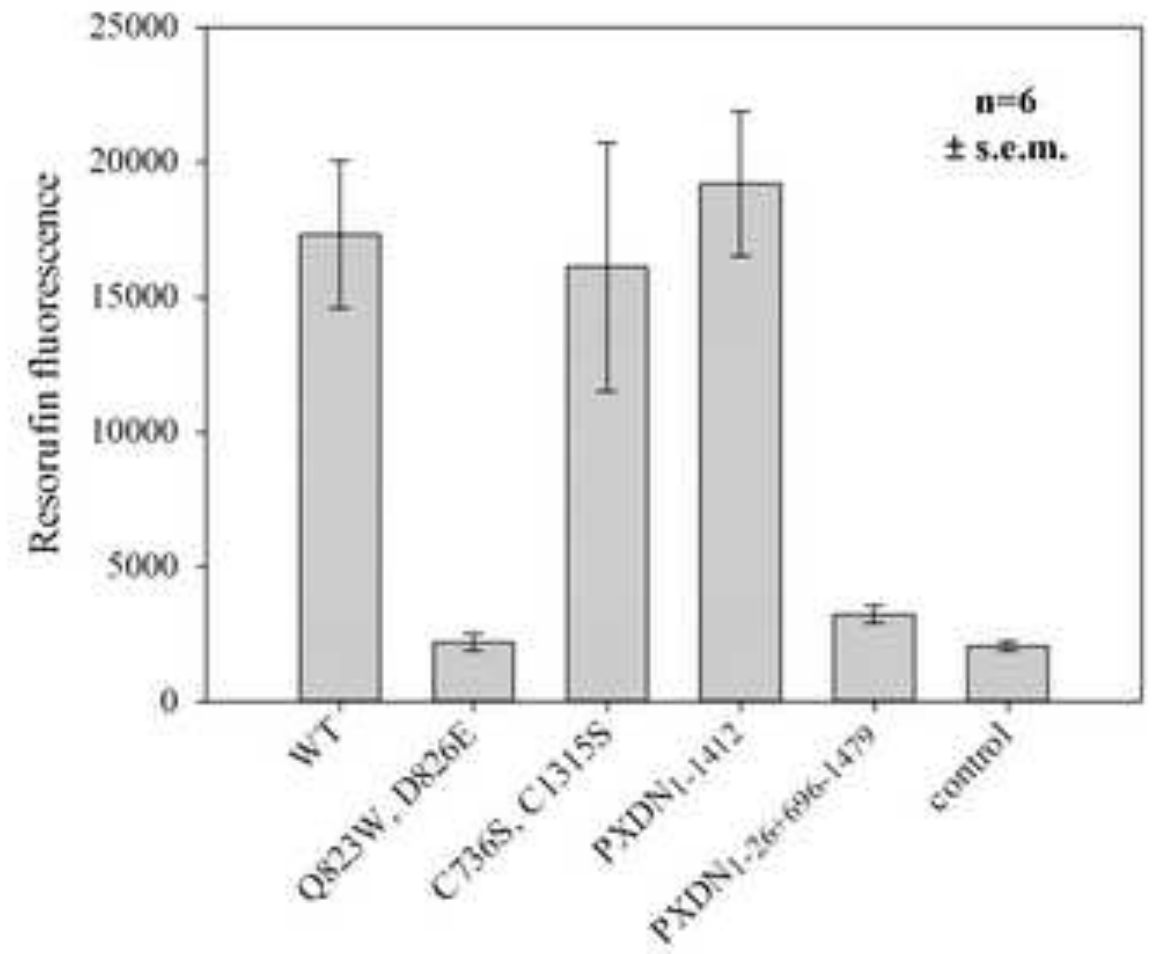

B

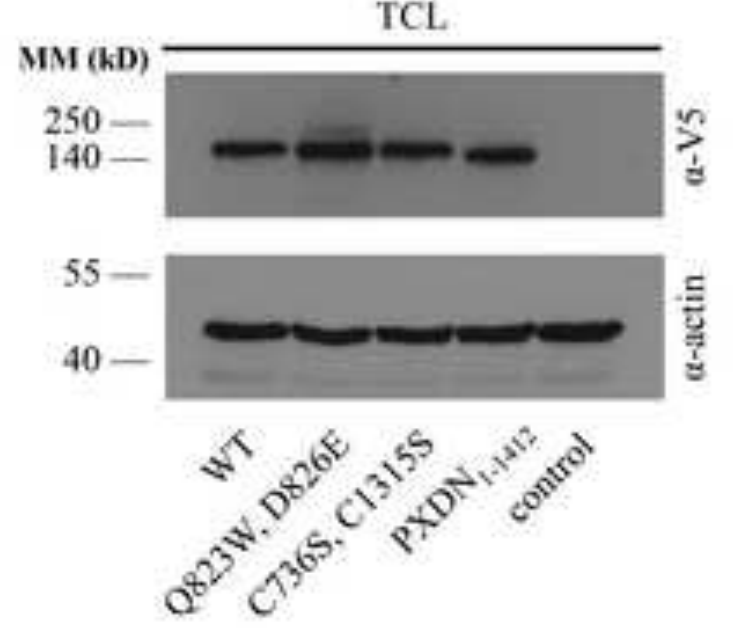

C

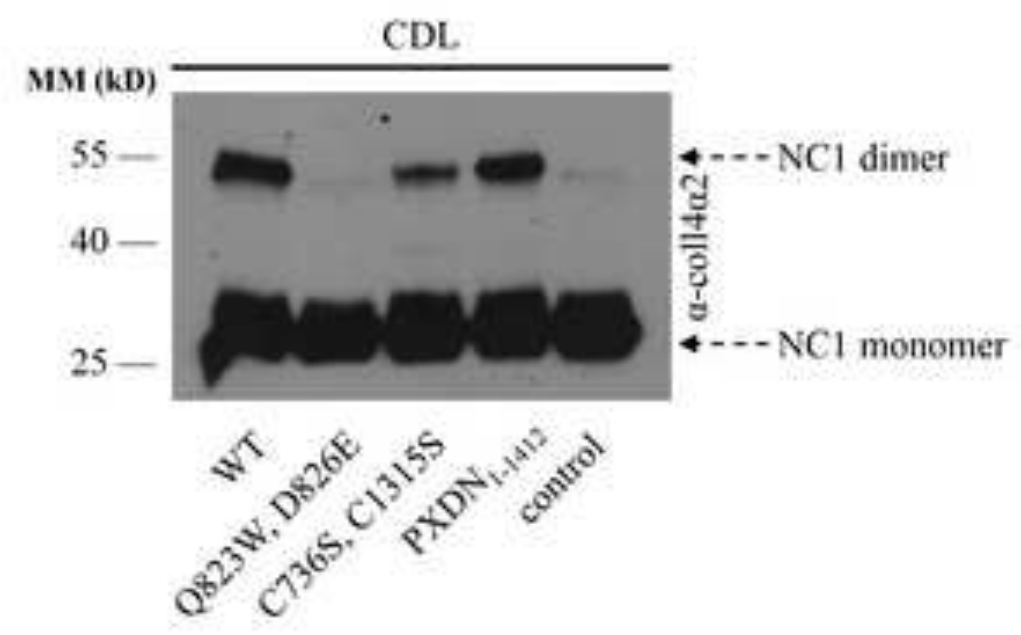



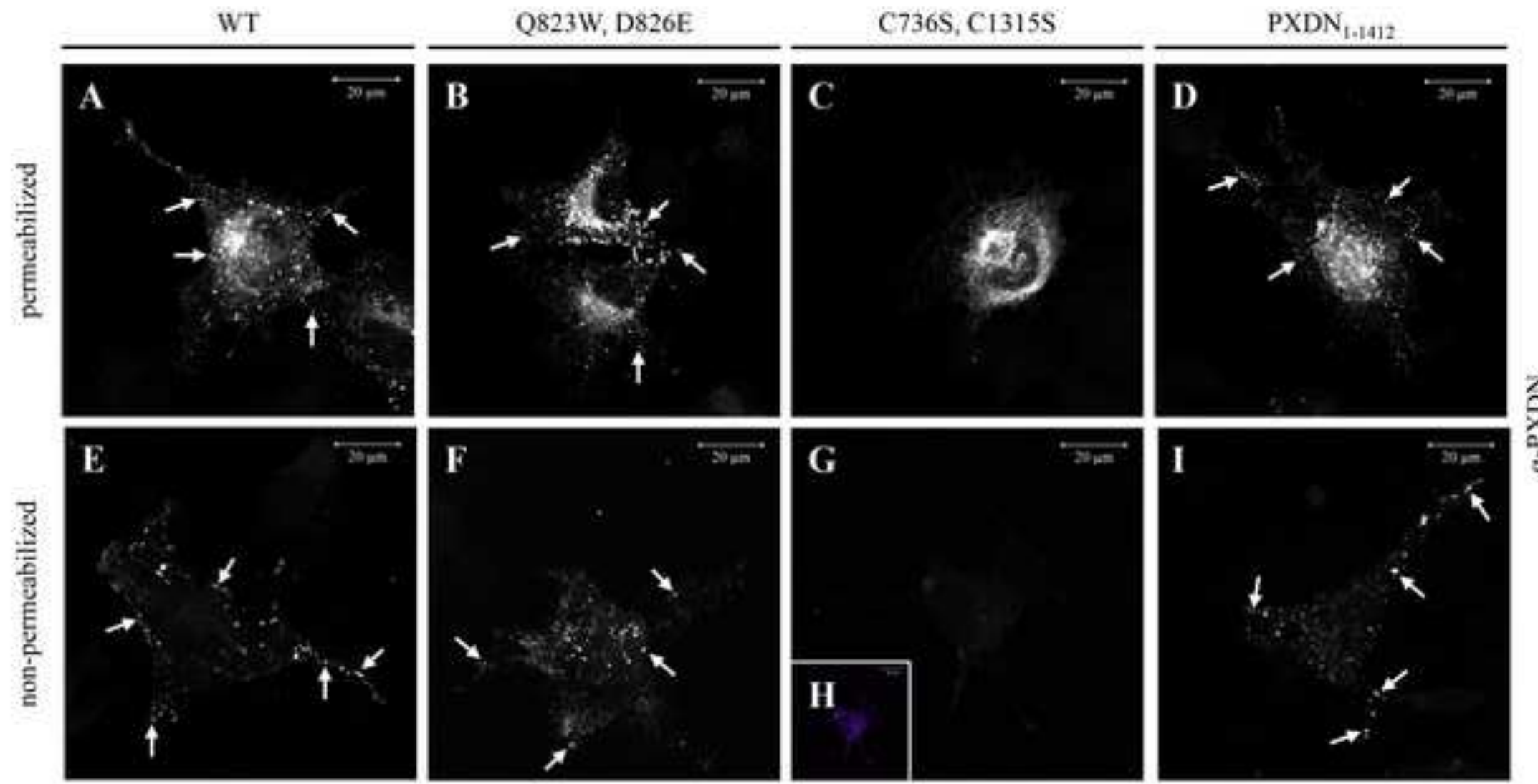


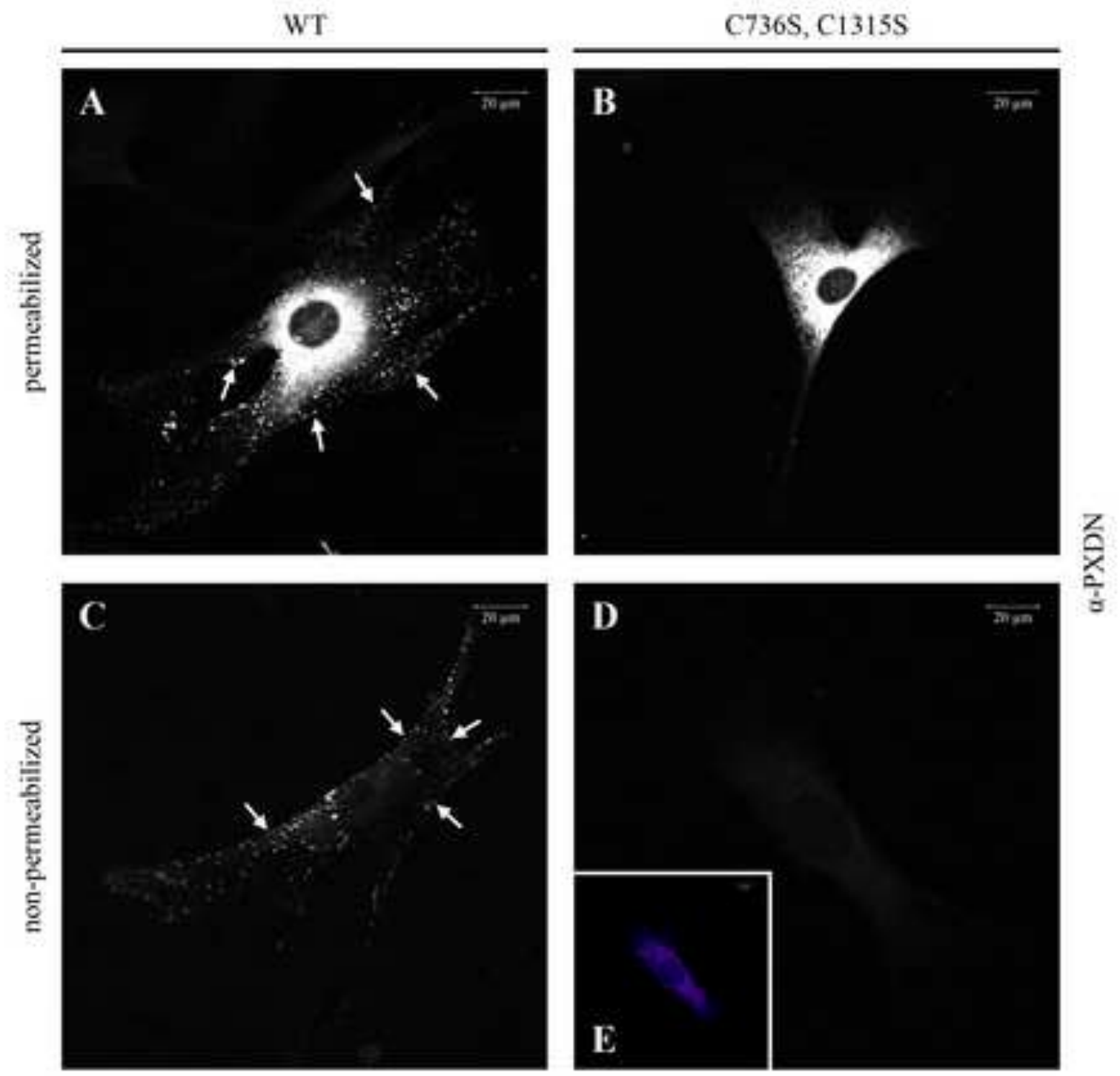


Supplementary Figure 1
Click here to download Supplementary Material: Suppfig1.tif

Supplementary Figure 1
Click here to download Supplementary Material: Suppfig1.tif

Supplementary Figure 1
Click here to download Supplementary Material: Suppfig1.tif

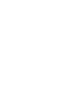

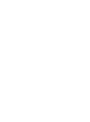

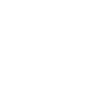

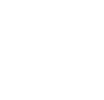

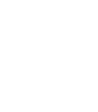

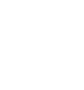

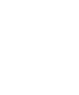

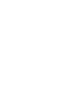
.

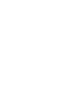

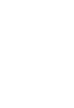

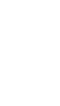

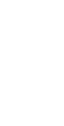
$+2$

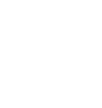

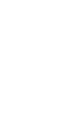

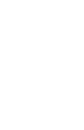

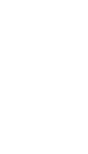

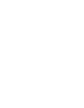

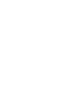

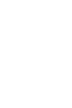

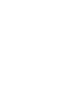

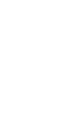

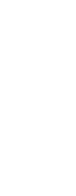

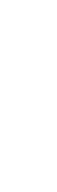

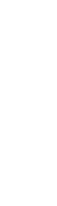

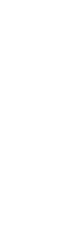

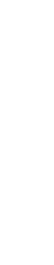
(1) 更

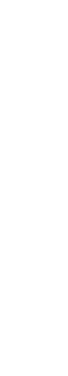

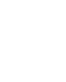
更

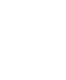

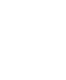

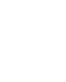

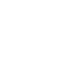

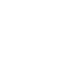

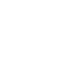

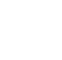


Supplementary Figure 2
Click here to download Supplementary Material: Suppfig2.tif

Supplementary Figure 2
Click here to download Supplementary Material: Suppfig2.tif a

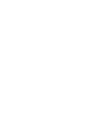

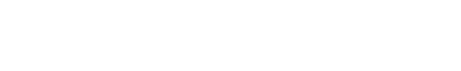

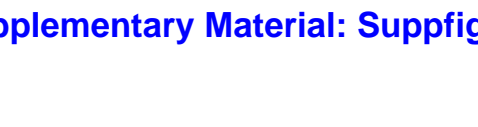


Supplementary Figure 3
Click here to download Supplementary Material: Suppfig3.tif

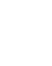

if

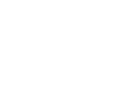

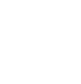


Supplementary Figure 4

Click here to download Supplementary Material: Suppfig4.tif

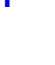

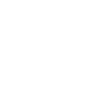

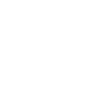

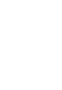

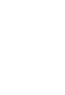

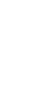

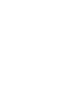
更

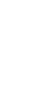

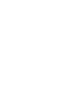

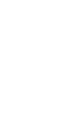
更

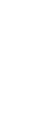

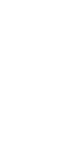
(n) (2)

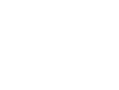

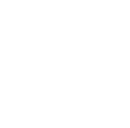

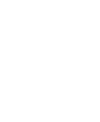

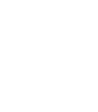

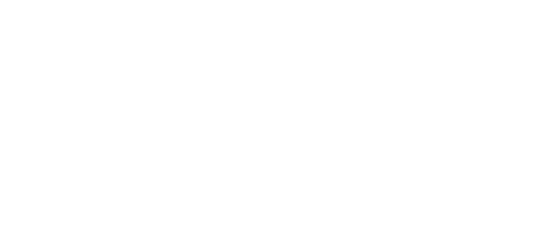

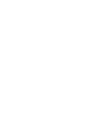
列

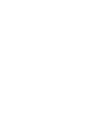

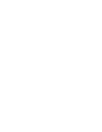

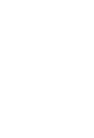

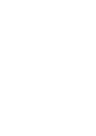

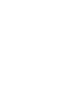

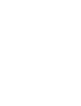

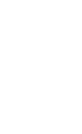

